\title{
Analytical integrate-and-fire neuron models with conductance-based dynamics and realistic PSP time course for event-driven simulation strategies Michelle Rudolph-Lilith*, Mathieu Dubois and Alain Destexhe
}

Address: UNIC, CNRS, 91198 Gif-sur-Yvette, France

Email: Michelle Rudolph-Lilith* - rudolph@iaf.cnrs-gif.fr

* Corresponding author

from Eighteenth Annual Computational Neuroscience Meeting: CNS*2009 Berlin, Germany. 18-23 July 2009

Published: 13 July 2009

BMC Neuroscience 2009, I0(SuppI I):P23 doi:I0.I I86/I47|-2202-I0-SI-P23

This abstract is available from: http://www.biomedcentral.com/I47I-2202/I0/SI/P23

(c) 2009 Rudolph-Lilith et al; licensee BioMed Central Ltd.

Recently, we developed models of analytical integrateand-fire (IF) neurons with conductance-based (COBA) dynamics, the gIF neuron models [1]. These models are based on an analytical approximation of the differential equation describing the IF neuron with exponential synaptic conductances and were successfully tested with respect to their response to random and oscillating inputs. Due to their simple mathematical structure, the gIF models are best suited for fast, event-driven simulation strategies. However, the approximations were based on the assumption of instantaneous rise and exponential decay of the postsynaptic potential (PSP). Consequent to this instantaneous rise, spikes were always produced at the onset of the PSP, which might have consequences for the temporal precision of the cellular responses, as the finite rise time of the PSP and the consequent "delay" between the onset of synaptic input and spike were not present in this model.

We addressed this limitation by conceiving an analytical approximation of the COBA IF neuron model with the full PSP time course, the gIF4 model. Such models are more complex to calculate because the spike does not occur at the PSP onset, but occurs later because of the finite PSP rise time and thus needs to be predicted in the future. We used a simple explicit analytical prediction of the threshold crossing to derive the spike time. In typical parameter regimes, both the PSP transient and spike time prediction deviate only a few percent from the predictions based on the numerical solution of the underlying differential equations. In high-conductance states, the error is smaller than $1 \%$. We compared the gIF4 dynamics to numerical simulations of the COBA IF model and the full Hodgkin-Huxley $(\mathrm{HH})$ neuron model. We characterized the responses with respect to output rate, coefficient of variation $(\mathrm{CV})$ and temporal precision. In all cases, excellent agreement in responses to random and oscillating inputs was observed, thus rendering the gIF4 model a faithful analytical approximation of COBA neuron models. Moreover, we tested the model in network simulations. Using randomly connected self-sustained networks with inhibitory and excitatory synapses, we faithfully reproduced the expected dynamical regimes and their borders when model parameters, such as the amplitude of synaptic conductances or the ratio between inhibitory and excitatory synapses, were varied. Finally, analyzing the gain in performance, the gIF4 model was more than two orders of magnitude faster than the HH model and still outperformed the COBA IF model by more than one order of magnitude.

In summary, the presented analytical gIF4 model provides an excellent approximation of COBA neuron models for use in efficient and precise event-driven simulations of large-scale neuronal networks with realistic, conductancebased synaptic interactions. 


\section{Acknowledgements}

Research supported by CNRS, the European Community (FACETS grant

FP6 I5879) and the ANR (HR-CORTEX).

\section{References}

I. Rudolph M, Destexhe A: Analytical integrate-and-fire neuron models with conductance-based dynamics for event-driven simulation strategies. Neural Comput 2006, 18:2146-2210.

Publish with Bio Med Central and every scientist can read your work free of charge

"BioMed Central will be the most significant development for disseminating the results of biomedical research in our lifetime." Sir Paul Nurse, Cancer Research UK

Your research papers will be:

- available free of charge to the entire biomedical community

- peer reviewed and published immediately upon acceptance

- cited in PubMed and archived on PubMed Central

- yours - you keep the copyright

Submit your manuscript here:

http://www.biomedcentral.com/info/publishing_adv.asp 